University of Nebraska - Lincoln

DigitalCommons@University of Nebraska - Lincoln

$1-14-2001$

\title{
Anisotropy-Induced Polarization Effects in Harmonic Generation by an Absorptive Medium
}

\author{
Bogdan Borca \\ Institute for Space Sciences, Bucharest, Romania \\ D. B. Milosević \\ University of Sarajevo, Bosnia and Herzegovina \\ Anthony F. Starace \\ University of Nebraska-Lincoln, astarace1@unl.edu
}

A. V. Flegel

Voronezh State University, Voronezh, Russia

M. V. Frolov

Voronezh State University, Voronezh, Russia

See next page for additional authors

Follow this and additional works at: https://digitalcommons.unl.edu/physicsstarace

Part of the Physics Commons

Borca, Bogdan; Milosević, D. B.; Starace, Anthony F.; Flegel, A. V.; Frolov, M. V.; and Manakov, N. L., "Anisotropy-Induced Polarization Effects in Harmonic Generation by an Absorptive Medium" (2001). Anthony F. Starace Publications. 117.

https://digitalcommons.unl.edu/physicsstarace/117

This Article is brought to you for free and open access by the Research Papers in Physics and Astronomy at DigitalCommons@University of Nebraska - Lincoln. It has been accepted for inclusion in Anthony F. Starace Publications by an authorized administrator of DigitalCommons@University of Nebraska - Lincoln. 


\section{Authors}

Bogdan Borca, D. B. Milosević, Anthony F. Starace, A. V. Flegel, M. V. Frolov, and N. L. Manakov 
Published in Super-Intense Laser-Atom Physics, edited by Bernard Piraux and Kazimierz Rzążewski. NATO Science Series:

Series II: Mathematics, Physics and Chemistry - Vol. 12. Dordrecht / Boston / London: Kluwer Academic Publishers, 2001.

Proceedings to the NATO Advanced Research Workshop on Super-Intense Laser-Atom Physics, Han-sur-Lesse, Belgium, September 24-30, 2000.

Copyright (c) 2001 Kluwer Academic Publishers. Used by permission.

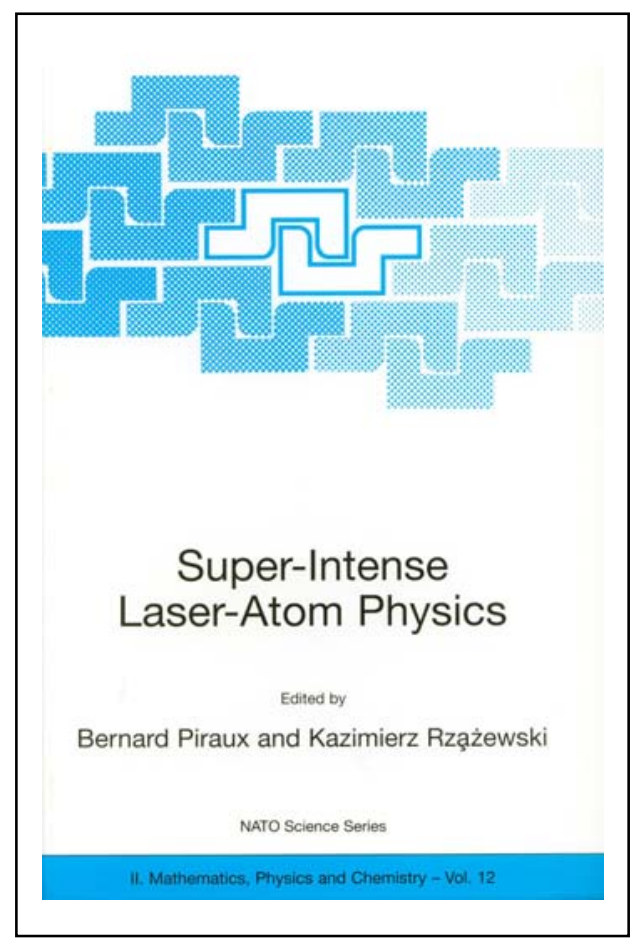




\title{
ANISOTROPY-INDUCED POLARIZATION EFFECTS IN HARMONIC GENERATION BY AN ABSORPTIVE MEDIUM
}

\author{
B. BORCA, ${ }^{*}$ D.B. MILOŠEVIĆ, ${ }^{+}$A.F. STARACE \\ Dept. of Physics and Astronomy, The University of Nebraska \\ 116 Brace Laboratory, Lincoln, NE 68588-0111, USA \\ AND \\ A.V. FLEGEL, M.V. FROLOV, N.L. MANAKOV \\ Voronezh State University, 394693 Voronezh, Russia
}

\section{Introduction}

High-order harmonic generation (HHG) has become a main topic of intense laser-atom physics [1]. Both to understand the HHG process better and to enhance its use in applications, many studies have focused on the control of HHG [1]. Among the most recent are those concerned with the polarization characteristics of the harmonics [2-8]. Other studies have focused on the use of a strong static electric field to control the intensities and plateau structure of HHG [9-12]. In this paper we present results of a general formulation of the problem of HHG in the case when the generating medium is anisotropic (see also [13]). Our specific results are for the case of anisotropy introduced by a strong, static electric field. However, we discuss also other ways of introducing anisotropy for which our analysis applies and for which similar results may be expected. We show that a static electric field has striking effects on the polarization of high-order harmonics and on the dependence of harmonic yield on the laser field ellipticity. We demonstrate that: (i) in the presence of a static electric field the harmonics are in general elliptically polarized, even for a linearly polarized driving laser (which contrasts with results obtained in the absence of a static field [5]), and (ii) the static electric field leads to a significant elliptic dichroism effect, i. e., the intensity of a harmonic differs substantially for right and left helicities of an elliptically polarized laser beam. We also discuss elliptic dichroism in the case when the generating medium is isotropic but a polarization sensitive detector is used for harmonic intensity measurements. All of these results 
demonstrate the possibility of significant control of polarization effects in HHG.

\section{Symmetry Analysis}

We consider a single atom interacting with a laser field of arbitrary polarization, i.e.,

$$
\mathbf{F}(\mathbf{r}, t)=F \operatorname{Re}\{\operatorname{eexp}[i(\mathbf{k} \cdot \mathbf{r}-\omega t)]\},
$$

where the complex polarization vector $\mathbf{e}$ is parametrized in a coordinateframe-invariant way using the laser field wave-vector $\mathbf{k}$ and the unit vector $\hat{\varepsilon}$ along the major axis of the laser polarization ellipse,

$$
\mathbf{e}=\frac{\hat{\varepsilon}+i \eta(\hat{\mathbf{k}} \times \hat{\varepsilon})}{\sqrt{1+\eta^{2}}},-1 \leq \eta \leq+1 .
$$

Here $\eta$ is the ellipticity, where $\eta=+1(-1)$ corresponds to right (left) circular polarization and $\eta=0$ to linear polarization. In terms of these vectors, the degree of linear polarization is given by $l=\mathbf{e} \cdot \mathbf{e}=\left(1-\eta^{2}\right) /(1+$ $\left.\eta^{2}\right)$, and the degree of circular polarization by $\xi=i \hat{\mathbf{k}} \cdot\left(\mathbf{e} \times \mathbf{e}^{*}\right)=2 \eta /\left(1+\eta^{2}\right)$, both of which are simply related to the usual Stokes parameters $S_{1}, S_{2}, S_{3}$ (i. e., $\xi=S_{2}, l=\sqrt{S_{1}^{2}+S_{3}^{2}}$ ) [14]. The static electric field $\mathcal{F}$ is oriented along the direction $\hat{\mathbf{e}}_{0}$, which we assume in the following to lie in the plane of the laser polarization ellipse, perpendicular to $\mathbf{k}$.

Complete information concerning the intensity and polarization properties of the $n$th harmonic may be extracted from the amplitude $A_{n \omega}\left(\mathrm{e}^{\prime}\right)$ describing dipole emission of a harmonic (with frequency $\omega^{\prime}=n \omega$, measured polarization $\mathbf{e}^{\prime}$, and propagation direction $\hat{\mathbf{k}}^{\prime}=\hat{\mathbf{k}}$ ) by an atom in the presence of fields $\mathcal{F}$ and $\mathbf{F}$, where

$$
A_{n \omega}\left(\mathbf{e}^{\prime}\right)=\mathbf{e}^{\prime *} \cdot \tilde{\mathbf{d}}_{n}
$$

and $\tilde{\mathbf{d}}_{n}$, defined in Eq. (9), is a (complex) matrix element of the dipole operator. It is useful at this point to specify what can be deduced from symmetry considerations alone, independent of any theoretical model for obtaining numerical results. Taking into account, as in [3], the spatial and temporal symmetry properties of the vectors involved [i.e., $\mathbf{F}(\mathbf{r}, t), \mathcal{F}$, e, $\mathbf{e}^{*}$, and $\left.\tilde{\mathbf{d}}_{n}\right]$ as well as the fact that only the part of $\tilde{\mathbf{d}}_{n}$ transverse to $\hat{\mathrm{k}}$ contributes to Eq. (3), one may express $\tilde{\mathbf{d}}_{n}$ in general as,

$$
\tilde{\mathbf{d}}_{n}=\chi_{1} \mathbf{e}+\chi_{2} \mathbf{e}^{*}+\chi_{3} \hat{\mathbf{e}}_{0} .
$$

The complex scalars $\chi_{1,2,3}$ are the nonlinear susceptibilities, which depend only on non-vector parameters (e. g., $\omega, F, \mathcal{F}, l$ ) and on the angle $\varphi$ between 
$\hat{\varepsilon}$ and $\hat{\mathbf{e}}_{0}$. Their values can be obtained by numerical calculation, based on a specific theoretical model. The total intensity of the $n$th harmonic, summed over its polarization states, is proportional to $\left|\tilde{\mathbf{d}}_{n}\right|^{2}$, where:

$$
\begin{array}{r}
\left|\tilde{\mathbf{d}}_{n}\right|^{2}=\sum_{i=1}^{3}\left|\chi_{i}\right|^{2}+\sqrt{2(1+l)} \operatorname{Re}\left[\left(\chi_{1}+\chi_{2}\right) \chi_{3}{ }^{*}\right] \cos \varphi \\
+\quad 2 l \operatorname{Re}\left(\chi_{1} \chi_{2}{ }^{*}\right)-\xi \sqrt{2 /(1+l)} \operatorname{Im}\left[\left(\chi_{1}-\chi_{2}\right) \chi_{3}{ }^{*}\right] \sin \varphi .
\end{array}
$$

The last term in Eq. (5), involving $\xi$, exhibits elliptic dichroism: it has opposite signs for right $(\xi>0)$ and left $(\xi<0)$ elliptic polarization of the laser field. Obviously this term vanishes for $\xi=0$ (linear laser polarization). It vanishes also for $|\xi|=1$ (circularly polarized laser light), but this is not obvious from $\mathrm{Eq}$. (5) and requires a detailed analysis of the $l$ and $\varphi$-dependence of the susceptibilities $\chi_{i}$, e. g., in terms of higher order perturbative expansions in $F$ (cf. [3]). Symmetry considerations imply that when $\varphi=\pi / 2$ the elliptic dichroism term also vanishes. Therefore, when $0<|\xi|<1$ and $0<\varphi<\pi / 2$, elliptic dichroism may be observed, caused by an interference between the real and imaginary parts of $\chi_{1,2}$ and the static-electric-field-induced component of the susceptibility tensor, $\chi_{3}$.

The polarization properties of the $n$th harmonic are described by its Stokes parameters $S_{i}^{n}, 1 \leq i \leq 3$ [14]. These are defined in terms of the intensity of the $n$th harmonic having a detected polarization $\mathbf{e}^{\prime}: I_{n \omega}\left(\xi^{\prime}, \theta\right) \propto$ $\left|A_{n \omega}\left(\mathbf{e}^{\prime}\right)\right|^{2}$, where $\theta$ is the angle between the directions $\hat{\varepsilon}$ and $\hat{\varepsilon}^{\prime}$ of the major axes of the polarization ellipses respectively of the laser and the detected harmonic. Thus $S_{1}^{n}$ equals the difference between $I_{n \omega}\left(\xi^{\prime}=0, \theta\right)$ for $\theta= \pm \pi / 4$ divided by the sum. Similarly $S_{2}^{n}$ involves $I_{n \omega}\left(\xi^{\prime}= \pm 1, \theta\right)$, and $S_{3}^{n}$ involves $I_{n \omega}\left(\xi^{\prime}=0, \theta\right)$ for $\theta=0, \pi / 2$. For a linearly polarized laser, Eq. (4) shows that there are only two independent susceptibilities, $\chi_{\|}=\chi_{1}+\chi_{2}+\chi_{3} \cos \varphi$ and $\chi_{\perp}=\chi_{3} \sin \varphi$. One easily finds that the harmonic's intrinsic degree of circular polarization, $\xi_{n}$, and offset angle, $\theta_{n}$, are:

$$
\begin{gathered}
\xi_{n} \equiv S_{2}^{n}=\frac{2 \operatorname{Im}\left(\chi_{\perp} \chi_{\|}^{*}\right)}{\left|\chi_{\|}\right|^{2}+\left|\chi_{\perp}\right|^{2}}, \\
\tan 2 \theta_{n} \equiv \frac{S_{1}^{n}}{S_{3}^{n}}=\frac{2 \operatorname{Re}\left(\chi_{\perp} \chi_{\|}^{*}\right)}{\left|\chi_{\|}\right|^{2}-\left|\chi_{\perp}\right|^{2}} .
\end{gathered}
$$

As for elliptic dichroism, the ellipticity $\xi_{n}$ originates from an interference of the real and imaginary parts of the nonlinear susceptibilities and may be observed for $0<\varphi<\pi / 2$. In contrast, the offset angle originates from the obvious anisotropy of the atom in a static field and is nonzero even for real 
$\chi_{\|}$and $\chi_{\perp}$. Note that each of the two dissipation-induced effects [Eqs. (5) and (6)] is described by a different combination of the $\chi_{i}^{\prime}$ 's: $\operatorname{Im}\left[\left(\chi_{1} \pm \chi_{2}\right) \chi_{3}^{*}\right]$ thus independent measurements of the two effects give complete information on the parameters which govern these effects: $\chi_{1} \chi_{3}^{*}$ and $\chi_{2} \chi_{3}^{*}$.

\section{Complex Quasienergy Approach for the Calculation of HHG}

The above symmetry analysis is independent of any dynamical model, but quantitative estimations of the magnitude of the effects require numerical calculations of the amplitudes in Eq. (3). An accurate, ab initio way to calculate them nonperturbatively is to use the quasistationary, quasienergy states (QQES) or non-Hermitian Floquet states (see, e. g., [15]) for an atom in strong laser and static electric fields, $\Phi_{\mathcal{E}}(\mathbf{r}, t)$, where $\mathcal{E}$ is the complex quasienergy. Because of their asymptotically divergent terms in $r$ (in the open ionization channels), the QQES wavefunctions are not normalizable in the standard way; thus matrix elements of the dipole emission operator $\mathbf{r}$ between QQES functions diverge when calculated in the usual way. Instead, properly normalized (dual) functions, $\tilde{\Phi}_{\mathcal{E}}(\mathbf{r}, t)$, must be used as bra vectors in a calculation of transition matrix elements $[16,17]$. In the presence of two fields, $\mathcal{F}$ and $\mathbf{F}(\mathbf{r}, t)$ with an arbitrary elliptical polarization, the proper dual functions are given by

$$
\tilde{\Phi}_{\mathcal{E}}(\mathbf{r}, t)=\left[\Phi_{\mathcal{E}}(\mathbf{r},-t ; \eta \rightarrow-\eta)\right]^{*}
$$

For $\eta=0$, the dual function in Eq. (8) coincides with that introduced by Potvliege and Shakeshaft [16] for a linearly polarized laser field. The time-dependent (complex) dual dipole moment is thus calculated as

$$
\tilde{\mathbf{d}}(t)=\left\langle\tilde{\Phi}_{\mathcal{E}}(\mathbf{r}, t)|\mathbf{r}| \Phi_{\mathcal{E}}(\mathbf{r}, t)\right\rangle=\frac{1}{2} \sum_{n} \tilde{\mathbf{d}}_{n} e^{-i n \omega t}
$$

using $\tilde{\Phi}_{\mathcal{E}}(\mathbf{r}, t)$ as the bra vector. The $n$th Fourier coefficient, $\tilde{\mathrm{d}}_{n}$, of $\tilde{\mathbf{d}}(t)$ is then used in $\mathrm{Eq}$. (3) for the generation amplitude.

Based on this QQES approach, we analyze the HHG amplitude using a 3-dimensional zero-range potential model for the atom. The QQES-solution for this model [18] has been used in recent HHG-calculations (see, e.g., $[19,9,10])$. We note that if (as done here) the quasienergy $\mathcal{E}$ is approximated by the unperturbed binding energy, $E_{0}$, of the model atom and if all but the leading Fourier-coefficients of $\Phi_{\mathcal{E}}(\mathbf{r}, t), a_{n}$, at the origin $(r \rightarrow 0)$ are neglected, as in [19], then our approximate QQES amplitude $A_{n \omega}\left(\mathbf{e}^{\prime}\right)$ coincides with that obtained in the so-called " $S$-matrix approach" $[7,10,20]$ provided that the latter takes into account the continuum-continuum terms [7]. Ref. [7] discusses the existence of two different definitions for the HHG 
amplitude, denoted by the terms "S-matrix" and "dipole-moment expectation value". In fact, the authors of [7] argued for the validity of the "dipole-moment expectation value" definition based on Ref. [19], where the expectation value of $\mathbf{r}$ was calculated in the QQES approach, without using $\tilde{\Phi}_{\mathcal{E}}(\mathbf{r}, t)$. As discussed above, this expectation value is divergent. (The divergence of the result in Ref. [19] for $n=1$ is explicitly shown in that paper, and, for higher $n$, divergences appear only if one calculates the HHG amplitude beyond the approximation $a_{n} \sim \delta_{n 0}$. However, this latter approximation is a good one for low frequencies $(\omega \ll 1)$ and for the intensities which are considered in that paper; therefore their numerical results are consistent with those of the "S-matrix" calculations.) The divergences inherent in the dipole expectation value imply therefore that it cannot be used for $a b$ initio calculations of HHG.

\section{Results and Discussion for the Zero-Range Potential Model}

In the zero-range potential model for calculations of HHG by an elliptically polarized laser in the presence of a static electric field, each susceptibility $\chi_{1,2,3}$ in Eq. (4) involves an infinite sum of one-dimensional time integrals of a product of Bessel functions. These integrals were evaluated numerically. In order to make our numerical results applicable to a variety of atoms and field parameters, we adopt scaled units: our energy unit is the atom binding energy $\left|E_{0}\right|$, and our electric field strength unit is the internal field, $F_{0}=\sqrt{2 m\left|E_{0}\right|^{3}} / e \hbar$. The displayed results are calculated for $\hbar \omega=0.2$, $F=0.2$, and $\mathcal{F}=10^{-3}$, all in scaled units, as a particular numerical example.

In Fig. 1 we predict elliptic dichroism for both even and odd harmonics as a function of the angle $\varphi$ between the directions of the major axis of the laser polarization ellipse, $\hat{\varepsilon}$, and the static electric field, $\hat{\mathbf{e}}_{0}$. If we denote by $I_{+}$and $I_{-}$the intensities obtained for laser ellipticities $\pm|\eta|$ then the dichroic ratio $\delta=\left(I_{+}-I_{-}\right) /\left(I_{+}+I_{-}\right)$, is a good measure of this effect. Our calculations show that significant values of $\delta$ appear as a result of either out-of-phase oscillations of $I_{+}$and $I_{-}$, or in-phase oscillations with, e. g., $\left|I_{+}\right|>\left|I_{-}\right|$. One sees from Fig. 1 that $\delta$ is significant for both even and odd harmonics, that it is significant throughout the plateau region, and that it is very sensitive to both $|\eta|$ and $\varphi$.

Figure 2 shows the circular polarization degree, $\xi_{n}$, for low-order even harmonics produced by a linearly polarized laser as a function of the angle $\varphi$ [cf. Eq. (6)]. For $\mathcal{F}=0$, a linearly polarized laser field generates only linearly polarized odd harmonics. As our static field strength is very small compared to the laser field strength, it doesn't change the polarization of the odd harmonics significantly (e. g., we found $\left|\xi_{n}\right|<0.03$ for $n=3,5,7$ ). 

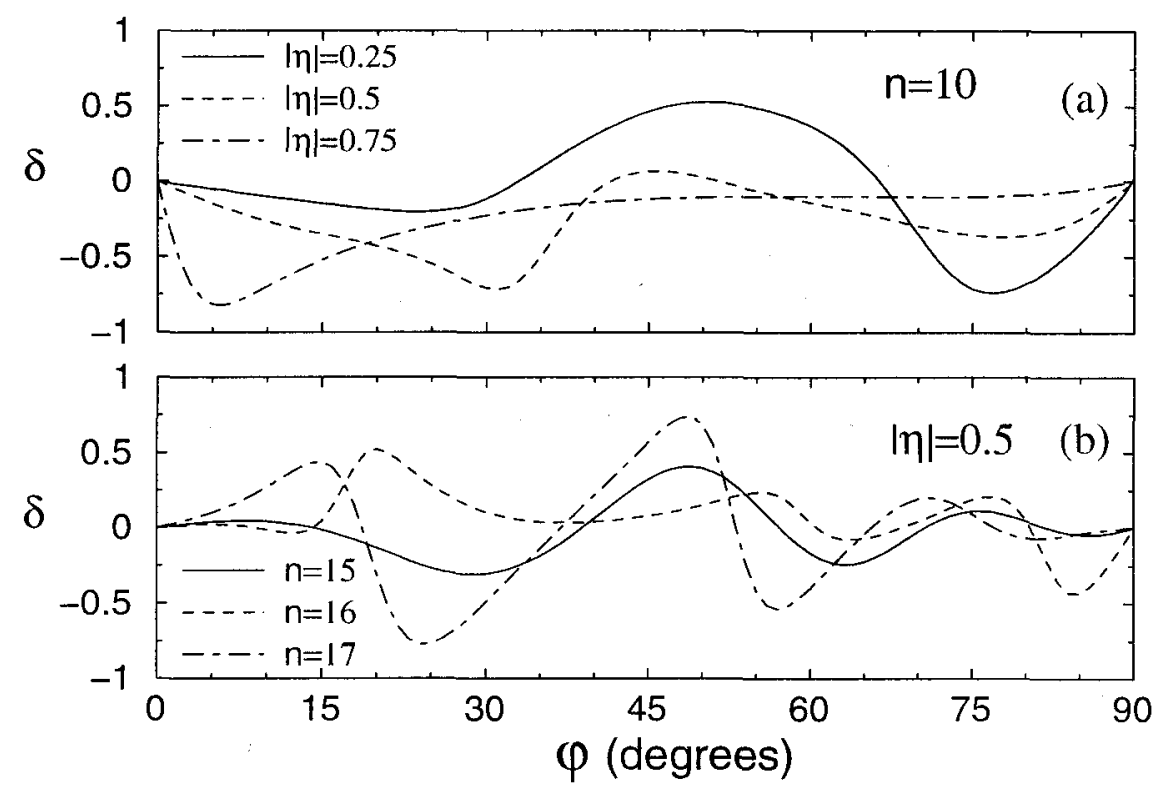

Figure 1. Elliptic dichroism parameter $\delta=\left(I_{+}-I_{-}\right) /\left(I_{+}+I_{-}\right)$for (a) $\mathrm{n}=10$ and $|\eta|=0.25,0.5$, and 0.75 , and (b) $n=15,16$, and 17 , and $|\eta|=0.5$. In scaled units (see text), $\omega=0.2, F=0.2$, and $\mathcal{F}=10^{-3}$.

However, the even order harmonics, which owe their presence to the static field, are strongly affected by its orientation. It is even possible to produce harmonics with polarizations that are very close to circular over a fairly large range of $\varphi$ [cf. Fig. 2 for $n=2$ and 6 and $60^{\circ} \leq \varphi \leq 70^{\circ}$ ]. As the harmonic order increases, the domain of significant non-linear polarization becomes narrower, and is increasingly shifted towards $\varphi=90^{\circ}$ (as one can see for the 8th harmonic). When $\varphi=90^{\circ}$, all harmonics are emitted with linear polarization but with different orientations: odd harmonics along $\hat{\varepsilon}$ and even ones along $\hat{\mathbf{e}}_{0}$.

Our analysis above [cf. (5) and (6)] shows that the polarization effects demonstrated in Figs. 1 and 2 stem from the anisotropy induced by the static field [cf. Eq. (4)] in an absorptive medium. We may extend this analysis by noting that the anisotropy can be induced by means other than a static electric field. For example, a similar effect may appear in harmonics generated by reflection of an intense laser beam by a metal surface, where the anisotropy is introduced by the surface normal vector. As another example, closer to the one we have treated, the required anisotropy may be introduced by a second, low-intensity, linearly-polarized laser beam collinear with the first, $\mathbf{F}_{\Omega}(t)=F_{\Omega} \hat{\mathbf{e}}_{0} \cos \Omega t$. In this case, if one considers only the harmonics of the high-intensity laser, Eqs. (3)-(7) have exactly the 


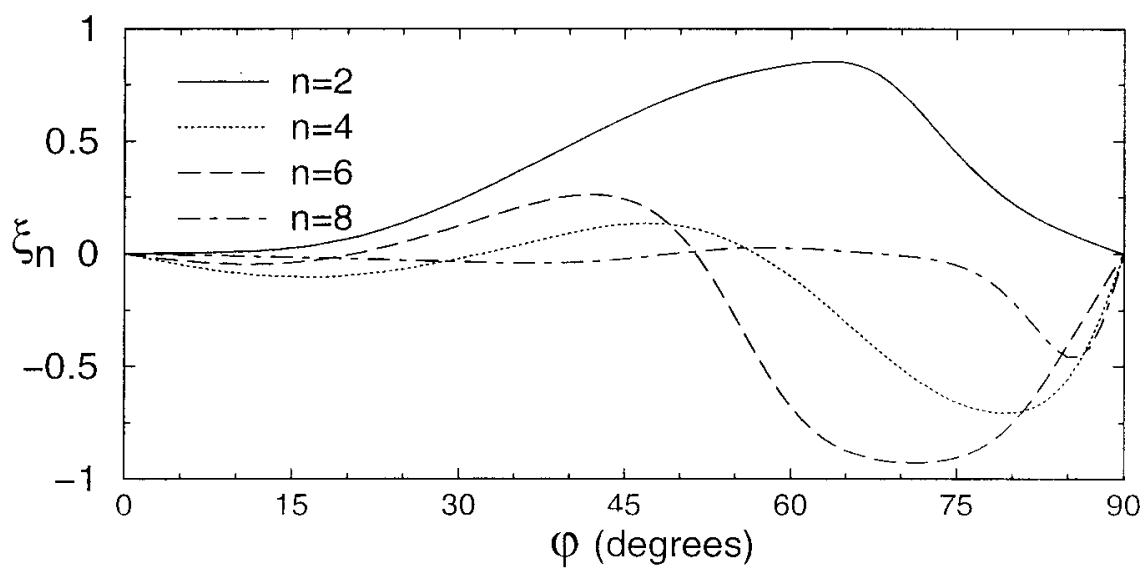

Figure 2. Circular polarization degree, $\xi_{n}$, for even harmonics $(n=2,4,6$, and 8) produced by a linearly polarized laser field for different angles $\varphi$. Parameter values $\omega, F$, and $\mathcal{F}$ are as in Fig. 1.

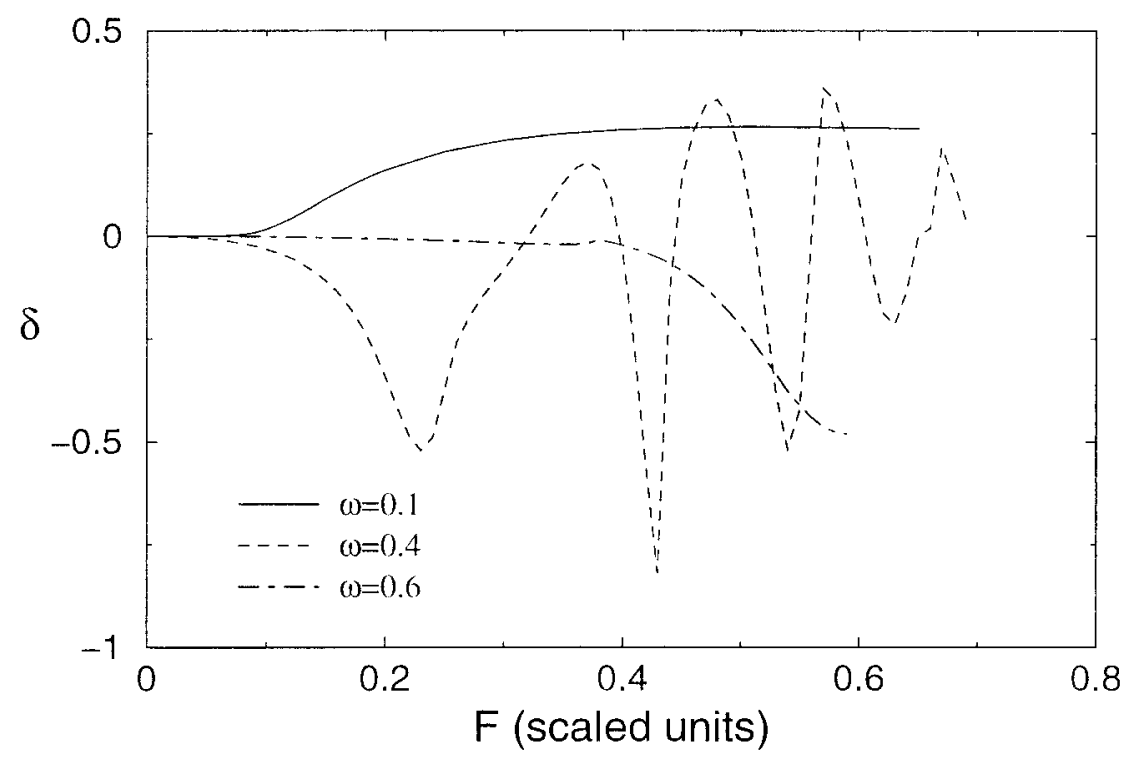

Figure 3. Elliptic dichroism in the case $\mathcal{F}=0$ for the third harmonic for three values of $\omega$. The laser ellipticity is $|\eta|=0.5$, the detector detects harmonics with $l^{\prime}=1$, and the angle between the laser polarization axis and the detector polarization axis is $\alpha=\pi / 4$.

same form and therefore lead to the same polarization effects. For a low frequency, $\Omega$, neglecting (in lowest approximation) the (weak) $\Omega$-dependence of the susceptibilities $\chi_{i}$, the only effect is to replace the static field arn- 
plitude $\mathcal{F}$ by an "effective amplitude" of order $F_{\Omega}$. Note that the effects predicted are invariant to spatial inversion of $\mathcal{F}$ (or $\mathbf{F}_{\Omega}$ ). As $\hat{\mathbf{e}}_{0} \rightarrow-\hat{\mathbf{e}}_{0}$, $\varphi \rightarrow \varphi+\pi$. This is equivalent in its effect on $\varphi$ to $\hat{\varepsilon} \rightarrow-\hat{\varepsilon}$. From Eqs. (1) and (2), $\hat{\varepsilon} \rightarrow-\hat{\varepsilon}$ is in turn equivalent to a phase change, $\omega t \rightarrow \omega t+\pi$, which does not affect the predictions (provided the two laser frequencies are incommensurate).

Finally, we note that elliptic dichroism appears also in HHG by an isotropic medium when the detector is polarization sensitive, detecting only photons having polarization $\mathbf{e}^{\prime}$. If $\alpha$ is the angle between the major polarization axis of the laser photons and the detector polarization axis (i. e., between the major axes of the polarization ellipses defined by $\mathbf{e}$ and $\mathbf{e}^{\prime}$ ), the intensity of the detected harmonic is given by [3]:

$$
\begin{aligned}
I_{n \omega}\left(\mathbf{e}, \mathbf{e}^{\prime}\right)= & \frac{1}{2}\left(\left|\chi_{1}\right|^{2}+\left|\chi_{2}\right|^{2}\right)\left(1+l l^{\prime} \cos 2 \alpha\right)+\frac{1}{2} \xi \xi^{\prime}\left(\left|\chi_{1}\right|^{2}-\left|\chi_{2}\right|^{2}\right) \\
& +\operatorname{Re}\left(\chi_{1} \chi_{2}^{*}\right)\left(l^{\prime} \cos 2 \alpha+l\right)+\xi l^{\prime} \operatorname{Im}\left(\chi_{1}^{*} \chi_{2}\right) \sin 2 \alpha,
\end{aligned}
$$

which clearly shows that the second and fourth terms are sensitive to the sign of the circular polarization degree, $\xi$, of the pump laser. The offset angle, $\theta_{n}$, i. e., the angle between the major axis of the polarization $e$ of the pump laser and that of the emitted harmonic, is given by:

$$
\tan \left(2 \theta_{n}\right)=\frac{2 \xi \operatorname{Im}\left(\chi_{1}^{*} \chi_{2}\right)}{2 \operatorname{Re}\left(\chi_{1}^{*} \chi_{2}\right)+l\left(\left|\chi_{1}\right|^{2}+\left|\chi_{2}\right|^{2}\right)} .
$$

The fact that the polarization of the emitted harmonics may be rotated with respect to that of the pump laser has been measured $[2,5]$. Note that, in the case $\mathcal{F}=0$, only one parameter governs the magnitude of both of these effects: $\operatorname{Im}\left(\chi_{1}^{*} \chi_{2}\right)$. This property was used in experimental studies in which the offset angle was extracted from dichroism measurements (see, e. g., Fig. 4 of [2]).

In Fig. 3 we present results for the elliptic dichroism parameter $\delta$ (as in Fig. 1) for the third harmonic for the case of a detector of linearly polarized photons oriented at an angle $\pi / 4$ with respect to the major axis of the elliptic polarization $(\eta=0.5)$ of the pump laser. One sees that $\delta \approx 0$ for $F<0.1$, which stems from the fact that $\operatorname{Im}\left(\chi_{1} \chi_{2}^{*}\right)$ is negligible until $F$ is of sufficient strength to begin depleting the initial state. One sees also from Fig. 3 that $\delta(F)$ is a very sensitive function of $\omega$.

Though we have presented our analysis and numerical results for the simplest conceptual case, that of HHG in the presence of a static field, we emphasize by the additional examples given above a major goal of our paper: to demonstrate how the introduction of a second polar vector in the problem [in addition to $\mathbf{F}(t)$ ] leads to interference between the complex susceptibilities $\chi_{i}$, the results of which are unusual polarization properties of the generated harmonics from an initially isotropic and absorptive 
medium. (This second polar vector may be a static electric field, the normal to a metal surface, a second linearly polarized laser field, or the orientation of a polarization-sensitive detector.) The predicted effects depend only on the magnitudes of the real and imaginary parts of the susceptibilities $\chi_{i}$. Physically, the imaginary parts are connected with the ionization of the target atom by the laser field, and hence they are zero if we neglect, this ionization. The polarization effects can therefore be called ionization(or dissipation-) induced effects. Note finally that the dichroic effects discussed above have a different physical origin from the well-known circular dichroism (which appears in chiral systems or magnetic solids), which is not dependent upon the existence of dissipation.

\section{Possibility of Experimental Verification}

Experimental verification of these harmonic polarization effects depends on the qualitative matching of our scaled parameters with a particular atomic system and a particular set of field strengths and frequencies. For example, for a Xe atom, the scaled parameters employed in Figs. 1 - 3 correspond to a laser with $\lambda=511.5 \mathrm{~nm}$ and intensity $2.48 \times 10^{14} \mathrm{~W} / \mathrm{cm}^{2}$, which are close to typical values for a frequency-doubled $\mathrm{Nd:YAG}$ laser $(\lambda=532 \mathrm{~nm})$. The static field strength, however, is $2.16 \mathrm{MV} / \mathrm{cm}$, which is far higher than typical laboratory static fields even though it is weak compared with the laser field $\left(\mathcal{F} / F=5 \times 10^{-3}\right)$. The requisite field strength ratio may nevertheless be achieved in a two color experiment in which the weak laser field, $\mathbf{F}_{\Omega}(t)$, has a much lower frequency, $\Omega$, than that of the intense laser. For example, the frequency of a $\mathrm{CO}_{2}$ laser (scaled frequency $\Omega=0.009$ for $\mathrm{Xe}$ ) is 22 times smaller than that of a frequency-doubled Nd:YAG laser, while the necessary $\mathcal{F} / F$ ratio could be achieved with an intensity of order $6 \times 10^{9} \mathrm{~W} / \mathrm{cm}^{2}$. The weak laser field may be regarded as approximately constant compared with the strong laser field and hence measured polarization results in such an experiment would be qualitatively similar to those predicted here for a laser plus static field configuration as long as the ratio of field strengths is similar.

\section{Conclusion}

The introduction of anisotropy into a generating medium (e. g., by means of a static electric field) permits significant control over the polarization properties of harmonics. Elliptic dichroism provides a unique case in the analysis of harmonic generation of measuring an effect which depends on the sign of the helicity of an elliptically-polarized laser beam. The predicted polarization effects allow the direct determination of the interference between real and imaginary (dissipative) parts of the nonlinear susceptibilities, which 
is useful for distinguishing between different models of ionization and harmonic generation by atoms in strong fields.

\section{Acknowledgments}

This work was supported by NSF Grant PHY-9722110, RFBR Grant 00-0217843 and INTAS Grant 97-0369 (AVF, MVF, and NLM), and the Alexander von Humboldt Foundation (DBM).

* On leave from Institute for Space Sciences, Bucharest-Măgurele 76900, Romania.

+ Present address: Faculty of Science, University of Sarajevo, Zmaja od Bosne 35, 71000 Sarajevo, Bosnia and Herzegovina

\section{References}

1. L. F. DiMauro and P. Agostini, Adv. At. Mol. Opt. Phys. 35, 79 (1995); P. Salières, A. L'Hullier, Ph. Antoine, and M. Lewenstein, ibid. 41, 83 (1999).

2. F. A. Weihe, S. K. Dutta, G. Korn, D. Du, P. H. Bucksbaum, and P. L. Shkolnikov, Phys. Rev. A 51, R3433 (1995).

3. N. L. Manakov, Zh. Eksp. Teor. Fiz. 110, 1244 (1996) [Sov. Phys.-JETP 83, 685 (1996)].

4. N. H. Burnett, C. Kan, and P. B. Corkum, Phys. Rev. A 51, R3418 (1995).

5. Ph. Antoine, B. Carré, A. L'Hullier, and M. Lewenstein, Phys. Rev, A 55, 1314 (1997).

6. M. Kakehata, H. Takada, H. Yumoto, and K. Miyazaki, Phys. Rev. A 55, R.861 (1997); D. Schulze, M. Dörr, G. Sommerer, J. Ludwig, P. V. Nickles, T. Schlegel, and W. Sandner, ibid. 57, 3003 (1998).

7. W. Becker, A. Lohr, M. Kleber, and M. Lewenstein, Phys. Rev. A 56, 645 (1997).

8. J. Gao, F. Shen, and J. G. Eden, J. Phys. B 32, 4153 (1999).

9. M. Q. Bao and A. F. Starace, Phys. Rev. A 53, R3723 (1996).

10. A. Lohr, W. Becker, and M. Kleber, Laser Phys. 7, 615 (1997).

11. B. Wang, X. Li, and P. Fu, J. Phys. B 31, 1961 (1998).

12. B. Wang, X. Li, and P. Fu, Phys. Rev. A 59, 2894 (1999).

13. B. Borca, A.V. Flegel, M. V. Frolov, N. L. Manakov, D. B. Milošević, and A. F. Starace, Phys. Rev. Lett. 85, 732 (2000).

14. See, e. g., $\$ 48$ of V. B. Berestetskii, E. M. Lifshitz, and L. P. Pitaievskii, Quantum Electrodynamics, 2nd ed. (Pergamon, Oxford, 1982).

15. S. I. Chu, Adv. At. Mol. Opt. Phys. 21, 197 (1985); N. L. Manakov, V. D. Ovsiannikov, and L. P. Rapoport, Phys. Rep. 141, 319 (1986).

16. R. M. Potvliege and R. Shakeshaft, in Atoms in Intense Laser Fields, ed. M. Gavrila (Academic, New York, 1992), p. 373; Phys. Rev. A 40, 3061 (1989).

17. N. L. Manakov, M. V. Frolov, A. F. Starace, and I. I. Fabrikant, J. Phys. B 33, R.141 (2000).

18. N. L. Manakov and A. G. Fainshtein, Zh. Eksp. Teor. Fiz. 79, 751 (1980) [Sov. Phys.-JETP 52, 382 (1980)].

19. W. Becker, S. Long, and J. K. McIver, Phys. Rev. A 50, 1540 (1994).

20. M. Yu. Kuchiev and V. N. Ostrovsky, J. Phys. B, 32, L189 (1999); Phys. Rev. A 60, 3111 (1999). 